\section{Who gets to be a PI?}

very three years, the IACUC at Great Eastern University met to review its own standard operating procedures (SOP) to assure they were up to date and compliant with federal regulations and policies. SOP III-42 stated that the Principal Investigator (PI) of an IACUC protocol is the person who has the responsibility and authority to direct the animal activities on the protocol. III-42 had always been interpreted by the IACUC as allowing postdoctoral scholars (postdocs) to function as PIs, as long as the postdoc's faculty mentor was listed on the protocol as the postdoc's sponsor. This interpretation was now being questioned by Dr. Jamie Matthews, an assistant professor of cell biology, who said that the SOP seemed to contradict itself. "How is it possible," she asked, "to be a PI with the responsibility of directing an animal research project if you have to have a faculty mentor overseeing or guiding you?" She wanted to know if a postdoc would have the authority to spend a mentor's grant money if, for example, the IACUC requested a pilot study or if a mentor's oversight would be required if a postdoc had a grant of his or her own. Matthews said that she wasn't trying to be a trouble maker; rather, she envisioned unnecessary research delays or animal welfare problems if a financial, personnel, or significant scientific problem arose and the mentor wasn't immediately available. Did the postdoc have or not have the authority to handle such problems?

Larry Covelli, the IACUC chairman, responded that the problems Matthews envisioned had never occurred during the many years he had chaired the IACUC, but if the committee thought that an SOP change was needed, it could vote for that change. However, as the discussion progressed it became clear that other members on the committee, especially the more senior faculty, were perfectly happy with the SOP as it was currently written because they saw the SOP as a good training mechanism for their postdocs and less work for them.

Do you think that Matthews has reasonable concerns? How do you think the Great Eastern IACUC should resolve the issue raised by Matthews?

\section{Jerald Silverman \\ University of Massachusetts Medical School, \\ Worcester, Massachusetts, USA. \\ e-mail: Jerald.Silverman@umassmed.edu}

Published online: 25 June 2018

https://doi.org/10.1038/s41684-018-0089-5

\title{
Determining PI status
}

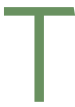

his scenario reviews the concept of who should be a Principal Investigator (PI) on an animal use protocol. It defines a PI as the person who has the responsibility and authority to direct the animal activities on a protocol. An IACUC member, Dr. Matthews, raises the question since Great Eastern University allows postdocs to be PIs, as long as the postdoc's faculty mentor is listed on the protocol as the postdoc's sponsor, how can the postdoc as the PI have the responsibility of directing an animal research project? Will the postdoc have the authority to direct and pay for a pilot study or deal with financial, personnel or significant scientific problems if they arose and the mentor was not available?

Dr. Matthews' concerns are valid in that the PI has many very important responsibilities as the leader of the research project and the signatory on the grant award. He/she sets the tone and assigns responsibilities to staff and collaborators. Publication of the final results is a central responsibility of PIs and so they are obliged to meet all requirements regarding publication and access to results. The PI is also required to comply with university policies as they relate to data security, confidentiality, data ownership, intellectual property, and copyright ${ }^{1}$. The PI is responsible for submitting an application to use animals in research, teaching or testing, as well as modifying or renewing existing research projects that use animals ${ }^{2}$. There must be assurance that the animal use section of any associated grant accurately reflects the animals used. The PI also ensures that the research staff is well trained on animal handling and the procedures outlined in the protocol, along with remaining current on techniques that help to reduce, replace or refine animal procedures ${ }^{2}$. The PI ensures that distress, discomfort or pain in the research animals is limited to that which is unavoidable in the conduct of valid scientific research or teaching and that the study is performed with due consideration for relevance to human or animal health, the advancement of knowledge, or the good of society ${ }^{2}$. The PI must make sure the animals are well cared for and that emergency veterinary care is administered to animals showing evidence of pain or illness, in addition to routine veterinary care, as prescribed for individual species. The PI understands that it is her/his responsibility to have available current emergency contact information for relevant personnel ${ }^{2}$.

Because of the numerous and sometime complex requirements to be a PI, it is suggested that postdocs along with graduate students, visiting scientists, and research associates only be allowed assignment as Co-Investigators under the direction of a PI. Individuals listed on animal use protocols as PIs should be of the rank of tenure track faculty, emeritus faculty, clinical faculty, scholar/scientist, or lecturer. These positions would have the ability to apply for and become funded for animal research projects and thereby would be able to assume supervisory, scientific, ethical and financial support for such a project. Postdoctoral fellows, graduate students, visiting scientists, and research associates would likely not be able to obtain significant funding and assume financial and ethical responsibility for an animal use protocol. The transition from student to responsible investigator occurs during this time and requires learning and being mentored. Individuals can and should assume supervisory, scientific, and ethical roles in research under the mentorship of faculty who have more experience in conducting animal studies and ultimately hold the ethical and financial responsibility for the study should any noncompliance violations occur requiring review by the IACUC or outside regulatory agencies. We also suggest that postdocs and others be actively involved in writing, modifying, and amending animal use protocols. The period during which a postdoc is mentored should be used to learn the role of PI, without the ultimate responsibility that a PI holds, so that they become well-trained, productive research scientists themselves.

Karen L. Rogers

Indiana University, Bloomington, Indiana, USA.

e-mail:rogerkar@iu.edu

Published online: 25 June 2018

https://doi.org/10.1038/s41684-018-0090-Z

References

1. Suckow, M. A. \& Yates, B. J. Research Regulatory Compliance. (Elsevier, Waltham, MA, 2015).

2. Institute for Laboratory Animal Research. Guide for the Care and Use of Laboratory Animals. 8th edn. (National Academies Press, Washington, DC, 2011). 